\title{
Emergency surgical management of cervical spine fracture-dislocation with acute paraplegia in COVID-19 (Coronavirus disease 2019)-suspected patient: first experience from a German spine centre
}

\author{
Mohamed Alhashash ${ }^{1,2} \cdot$ Walaa Elsebaiy ${ }^{3} \cdot$ Mohamed Farag $^{1} \cdot$ Mootaz Shousha $^{1,2}$
}

Received: 4 May 2020 / Revised: 15 September 2020 / Accepted: 3 October 2020 / Published online: 23 October 2020

(c) Springer-Verlag GmbH Germany, part of Springer Nature 2020

\begin{abstract}
Purpose We present an organized hospital plan for the management of Coronavirus disease (COVID-19) patients requiring emergency surgical interventions. To introduce a multidisciplinary approach for the management of COVID-19-infected patients and to report the first operated patient in the Corona unit.

Methods A detailed presentation of the hospital plan for a separate Corona unit with its intensive care unit and operating rooms. Description of the management of the first spine surgery case treated in this unit.

Results The Corona unit showed a practical approach for the management of an emergency cervical spine fracture-dislocation with acute paralysis. The patient is 92 -year-old female. The mechanism of injury was a simple fall during the stay in the internal medicine department where the patient was treated in the referring hospital. The patient had no other injuries and was awake and oriented. The patient did not have the clinical symptom of COVID-19, and the test result of COVID-19 done in the referring hospital was not available on admission in our emergency room. Education of the medical staff and organization of the operating theatre facilitated the management of the patient without an increased risk of spreading the infection. Conclusions The current COVID-19 pandemic requires an extra-ordinary organization of the medical and surgical care of the patients. It is possible to manage an infected or a potentially infected patient surgically, but a multidisciplinary plan is necessary to protect other patients and the medical staff.
\end{abstract}

Keywords COVID-19 $\cdot$ Emergency $\cdot$ Cervical fracture $\cdot$ Corona ward $\cdot$ Surgical planning

\section{Introduction}

\section{Coronavirus disease 2019}

In late 2019, a series of severe acute respiratory syndrome (SARS) was reported in China. The causative organism was detected, and the term Coronavirus disease 2019 (COVID19) was introduced $[1,2]$. A few weeks later, the number of cases increased dramatically. Cases outside China were

Mohamed Alhashash

hashmf1@yahoo.com

1 Department of Spine Surgery, Zentralklinik Bad Berka, Robert-Koch-Allee 9, 99437 Bad Berka, Germany

2 Department of Orthopedic Surgery, Alexandria University, Alexandria, Egypt

3 Department of Anesthesia and Intensive Care, Alexandria University, Alexandria, Egypt detected [3]. Confirmation of human-to-human transmission leads to the application of specific measures to reduce the rate of infection and thus to allow medical systems to manage the exponential demand on intensive care beds for such patients. By 11 March 2020, as the number of countries involved reached 114 with more than 118,000 cases and over 4000 deaths, the WHO declared the pandemic status [4].

\section{Start of national measures in Germany}

On Monday, 27 January 2020, a Chinese woman from Shanghai informed the company she was working for in Bavaria about her Coronavirus infection. Her German colleague was then tested positive. He was the first confirmed case in Germany [5]. Starting from this day, the national authorities in Germany started a general plan to prepare the medical system for a dangerous situation $[6,7]$.

First, the hospitals were prepared to accommodate many seriously ill Corona patients under high pressure. Both the 
government's activities to slow the spread and the preparation of the hospitals for many intensive care patients with severe illnesses were aimed at ensuring the best possible care for every ill person. Before the Corona crisis, there were 28,000 intensive care beds nationwide in Germany, including 20,000 with ventilation. These were occupied on average of 70-80 percent. The intensive care capacity is divided into intermediate care units (IMC) where most patients are not ventilated and mainly for peri-operative care, and patients with a medical condition not suitable for a peripheral station. Most IMC beds can be converted into ventilation units as the ventilators are available. The second category is the intensive care units (ICU), where all beds are equipped with ventilators. Before the pandemic, patients in both IMC and ICU were not all on ventilators [8].

Capacities were then expanded nationwide. Efforts were made in all hospitals, supported by central measures taken by the Federal Ministry of Health, to create further ventilation places in the medium term and to supply additional ventilation devices. The number of intensive care beds did increase to 40,000 and the ventilation places to 30,000 .

Second, the elective surgical procedures were rescheduled to allow hospitals to manage the expected increase in the number of COVID-19 patients and to avoid the postoperative increased risk of infection, especially after major operative interventions with reduced postoperative immune system response.

Third, educational and practical measures were undertaken in each hospital, including information and training of employees, the examination of stocks and stockpiling with personal protective equipment, pharmaceuticals and disinfectants. Besides, detailed information for patients and visitors and travelling history for each person, together with the expansion of COVID-19 tests, were further measures undertaken.

The German Interdisciplinary Association for Intensive Care and Emergency Medicine (DIVI), the Robert Koch Institute (RKI) and the German Hospital Society (DKG) have activated together with the DIVI intensive care register. This website registers and queries for free ventilation places in all clinics in Germany to optimize the cooperation between the hospitals and to provide a real overview of the intensive bed situation in the nation [9-11].

In our hospital, we have an intermediate care unit (IMC) with a capacity of 40 beds. The IMC is divided into a surgical IMC for peri-operative care and a medical IMC for cardiac and pulmonary patients that are medically not suitable for a peripheral station. Almost all patients in the IMC are not on a ventilator, but 30 beds are equipped with a ventilator. Besides, there are 35 beds in the intensive care unit (ICU). These beds are all equipped with a ventilator. This explains the high ventilation capacity in the German medical system. Stopping the elective operation resulted in a very rapid increase of the IMC capacity within one or two days as most postoperative patients leave the IMC within the first $24 \mathrm{~h}$ postoperatively.

\section{Methods}

\section{Changes in spine surgery practice}

In our hospital, the spine surgery department has rescheduled all elective operations. The surgical intervention was limited to four categories of spine disorders. The first group consisted of patients with unstable spine fractures. The second group included patients presenting with acute paralysis due to spinal canal stenosis or disc prolapse. The third category was spinal oncology, and the fourth group was spondylodiscitis patients presenting with sepsis or neurological deficits. Outpatient visits were limited to patients with a planned first postoperative examination or patients presenting with acute spine disorders or intractable symptoms. The waiting area for the outpatient clinic was modified to prevent close contact between patients, and the appointments were terminated to limit the waiting time before meeting the treating doctors. In addition, all visiting patients were asked about their travel history as well as about the presence of any respiratory symptoms or fever. All these measures aimed to reduce the local spread of infection among our patients as well as to protect our medical staff.

\section{Corona department}

The hospital crisis team developed an emergency plan for patients with COVID-19. A separate emergency room with a separate entrance was organized (the Corona emergency room). An isolation station was further prepared for positively tested patients. This station consisted of two units: one with standard isolation rooms, and the other consisted of isolation intensive care beds. This isolation station was connected to two operating theatres for emergency surgical interventions. These Corona theatres were equipped with an operating microscope, as well as two C-arms. Suspected cases waiting for the result of the PCR test were admitted in the Corona station but separately isolated rooms.

A well-defined strategy for patients' referral was then established. Any hospital seeking patient transfer to our hospital was asked to perform a PCR COVID test within $48 \mathrm{~h}$ before transmission, to admit the transferred patient at the right place in our hospital. With a negative test, patients were admitted to the ordinary hospital departments. In the case of a positive test, patients were admitted to the Corona unit.

An emergency referral was addressed in a different way. Emergency cases were defined to be patients with 
neurological deficits, sepsis or unstable spine. These patients were transferred without the COVID-19 test. The test was then performed on arrival, and the patients were operated when needed in the Corona theatre. All of them were managed as Corona positive in the special station for suspected cases till otherwise proved.

\section{Staff protecting measures}

The protection of the staff is a priority to maintain adequate continuous medical service for the patients. In our hospital, we passed two phases; the first phase was at the beginning of the pandemic. Persons who were on vacation or visited a risk zone and have no symptoms were instructed to stay 14 days in home isolation and can start working when staying symptom-free. People who have respiratory symptoms are immediately tested and were instructed to avoid working until the results were available. In the first three weeks, we did not have any positive cases in our staff.

The second phase started after the organization of the Corona unit. The medical staff is educated to be able to deal with infected or potentially infected patients. The staff is instructed to enter the hospital through a separate entrance. Protective medical supplies like masks and eye protection tools are available. The medical staff and the service person should wear a mask during the whole duty time. The hospital restaurant and café were closed. The gathering is limited to the minimum, and all should wear a mask. Meetings and communications between different departments were converted to video conferences or telephone meetings. Persons with any respiratory symptoms are immediately tested for COVID-19 and are not allowed to work until the results are available. Any medical staff that had contact with a positive person outside the hospital was instructed to have 14 days of home isolation.

\section{Patients protecting measures (Fig. 1)}

On admission, the COVID-19-positive patients are directed to the Corona ER and then to the separate isolation rooms or ICU isolation rooms. Patients should wear a protective mask during the stay outside the isolation rooms for any diagnostic procedure. The pathway in the hospital is planned, and the staff follows an exact route for connection between the different diagnostic and intervention rooms in the hospital. The main concern is the Corona-suspected patients as they may get the infection in the hospital. The suspected patients are managed in two ways, first as a source of infection. The personal measures are taken to avoid transmission to other patients and our staff. Second, the patient should be protected from the confirmed COVID-19-positive patients. The isolation rooms are separated in a way to prevent any contact between patients. The medical staff is not allowed to enter the isolation unit of different patients without a complete change of the protective measures, and proper disinfection must be done between every patient. Once the COVID-19

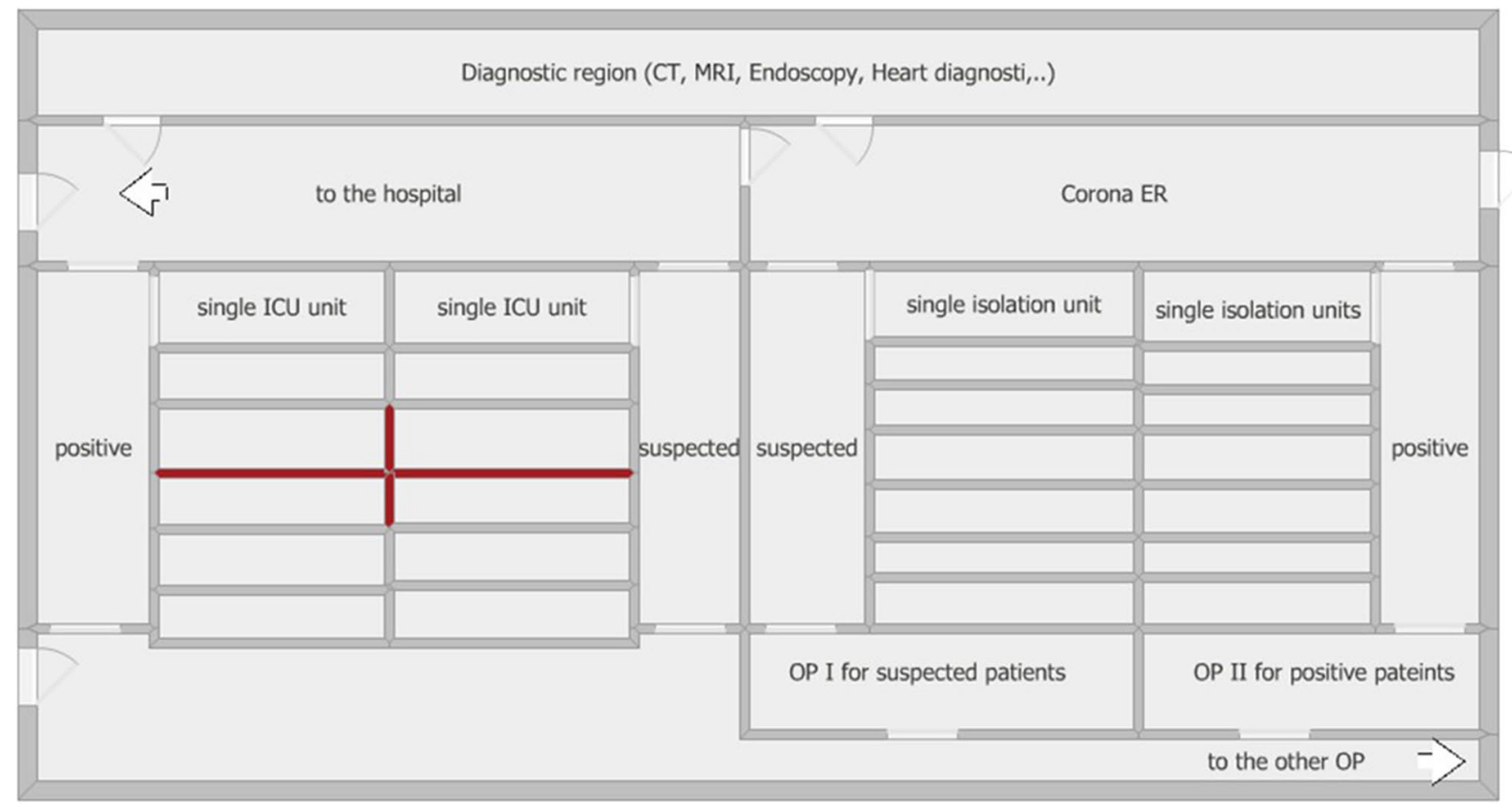

Fig. 1 The construction of the Corona unit with the separate isolation rooms and ICU units for confirmed positive cases and suspected cases. The two Corona operation rooms are connected to the unit 
test is available (12-24 h), the patient is transferred to a suitable place in the hospital. Family visits are prohibited for all patients during the hospital stay with the exception of terminal patients and patients in the palliative oncology department, and the visitors should be symptom-free and wear a protective mask.

In this report, we are presenting the first spine emergency surgical intervention in a patient with suspected COVID-19.

\section{Results}

\section{Case presentation (Fig. 2)}

On 22 April 2020, a 92-year-old female patient was referred to our emergency department with acute paraplegia after cervical spine trauma. The trauma was in the form of falling during her hospital stay in the internal medicine department in the referring hospital. The patient was acutely referred to our hospital before the result of the COVID-19 test was available.

We considered the case as suspected COVID-19. The patient was immediately admitted to the Corona emergency room two hours after trauma. On admission, the patient had a rigid cervical spine collar and was awake with Glasgow coma scale 15. The neurological examination showed incomplete paraplegia below the neurological level of C6 (ASIA B). Before the trauma, the patient was independent, and she had no significant associated medical disease. CT scan was performed in the referring hospital, and cervical spine MRI was immediately performed in the emergency department (Fig. 1). A traumatic fracture-dislocation C5-6 with spinal cord oedema was diagnosed. As the patient had an unstable fracture with acute paralysis, the decision for an emergency surgical intervention was made [12]. A posterior open reduction and stabilization combined with anterior decompression and fusion were planed.

\section{Modifications in the Corona operating rooms}

\section{Before surgery}

The Corona unit has two equipped operating rooms. One room is reserved for suspected patients, and the other room is for the confirmed cases. Measures are taken to prevent unnecessary movement of the staff. The instruments are prepared adequately to avoid the need for springing between rooms. Protective masks (FFP3 filtering facepiece particle

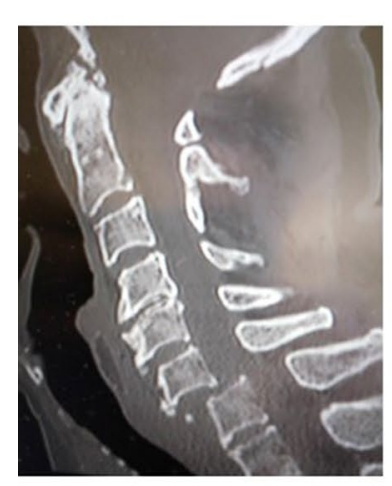

A

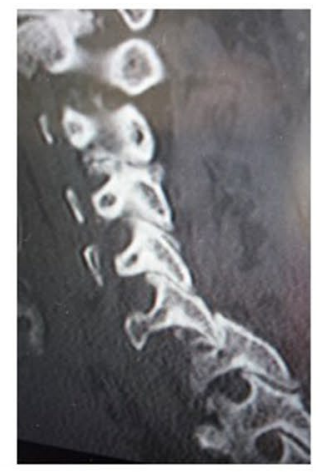

B

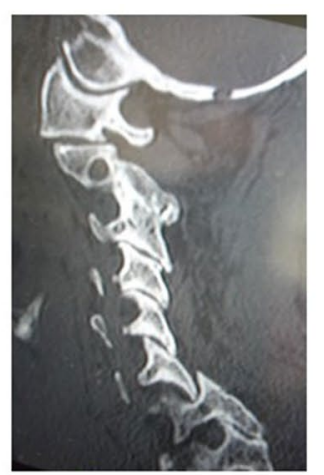

C

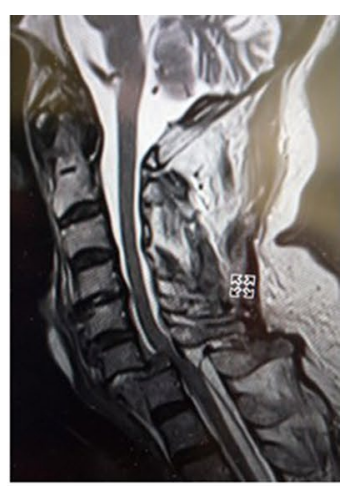

D

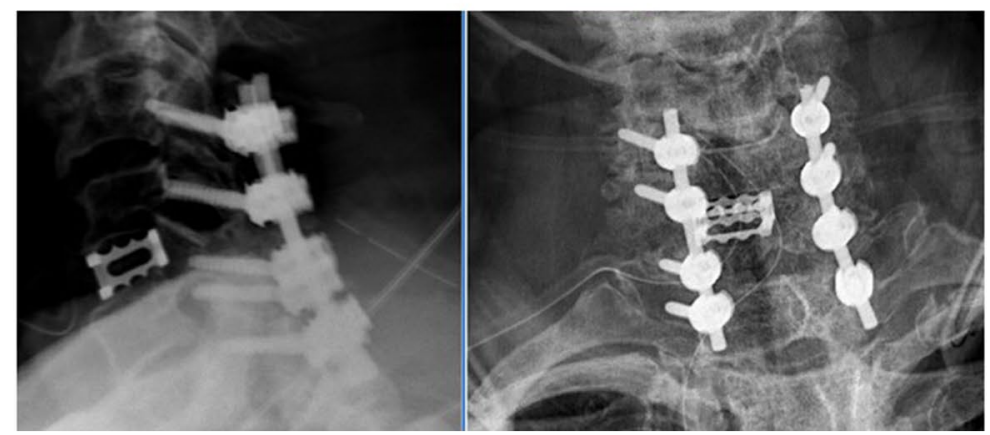

E

Fig. 2 A 92-year-old female patient presenting with fracture-dislocation C6-7. Sagittal CT a, facet joint dislocation on the right side b, facet joint dislocation on the left side $\mathbf{c}$, MRI examination $\mathbf{d}$ and postoperative X-ray showing reduction and fixation $\mathbf{e}$ 
3 ), head covers and eye goggles are adequately stocked in the operating rooms. The medical staff is trained for the proper dealing with the infected material during the operation. The number of persons in the operating room is reduced to a minimum. Patients are transferred to the Corona operating rooms under strict hygienic measures wearing a surgical mask and eye protection.

\section{During intubation and surgery (Fig. 3)}

The anaesthesia staff wears the same protective measures as the surgical staff. During intubation, only the staff involved in the procedure are allowed to enter the operating room. Endotracheal intubation, guided by a video laryngoscope with a disposable cover, is recommended. Disconnection of the patient for positioning should, when possible, be avoided. When disconnection is necessary, the ventilator filter must remain connected to the tube on the patients' side to avoid the dissemination of infection. After intubation, a wet compress covers the patient's face to reduce the possibility of infection transmission. The surgical team should reduce the use of cauterization and use the suction system properly. Extubation should be performed in the operating room and under the same measures used during intubation. The patient should wear a protective mask as soon as possible after the extubation.

\section{After surgery}

The surgical and anaesthesia staff are trained to take off the protective measures under the strict hygienic procedure. Additional help through another person is necessary to avoid contact with the infected masks, goggles and gowns during the removal of the protective material. The used instruments are separately sterilized. The potentially infected materials used during the operation, including surgical drapes, gowns, gloves and masks, were collected in separate trash with a double closure for proper disposal. The body fluids and the tissue material of the operated patients are also eliminated in the same way. After the procedure, all filters in the anaesthesia machine are removed and changed. The room is appropriately webbed and disinfected. The service persons are trained to deal with the infected materials and to perform the proper postoperative disinfection. The operating room remains closed for a minimum of four hours after the surgical procedure to reduce the possibility of infection transmission for the next patient. Patients with confirmed COVID-19 test stay in the Corona isolation unit until the improvement of the clinical symptoms and two negative COVID-19 tests $48 \mathrm{~h}$ apart. The maximum isolation time for a suspected patient is until the test results are available in 12 to $24 \mathrm{~h}$.

\section{Operative profile of the presented case}

The time between hospital admission and operation was $135 \mathrm{~min}$. The posterior approach with open reduction of the facet joint dislocation, posterior decompression and lateral mass screw fixation took $115 \mathrm{~min}$. Anterior discectomy C6/7 with decompression and cage augmented fusion took $40 \mathrm{~min}$. Operative blood loss was $450 \mathrm{ml}$ (300 $\mathrm{ml}$ posterior and $150 \mathrm{ml}$ anterior). There were no intraoperative complications. The patient was extubated in the operation theatre under strict hygienic measures and then was transferred to the ICU of the Corona unit. Postoperatively, no change in

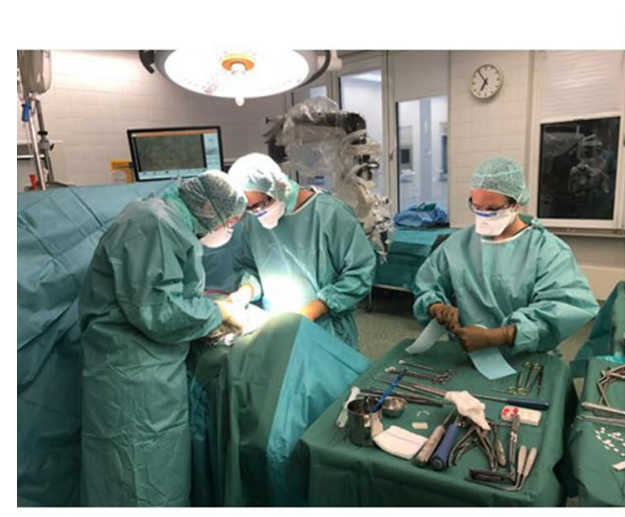

A

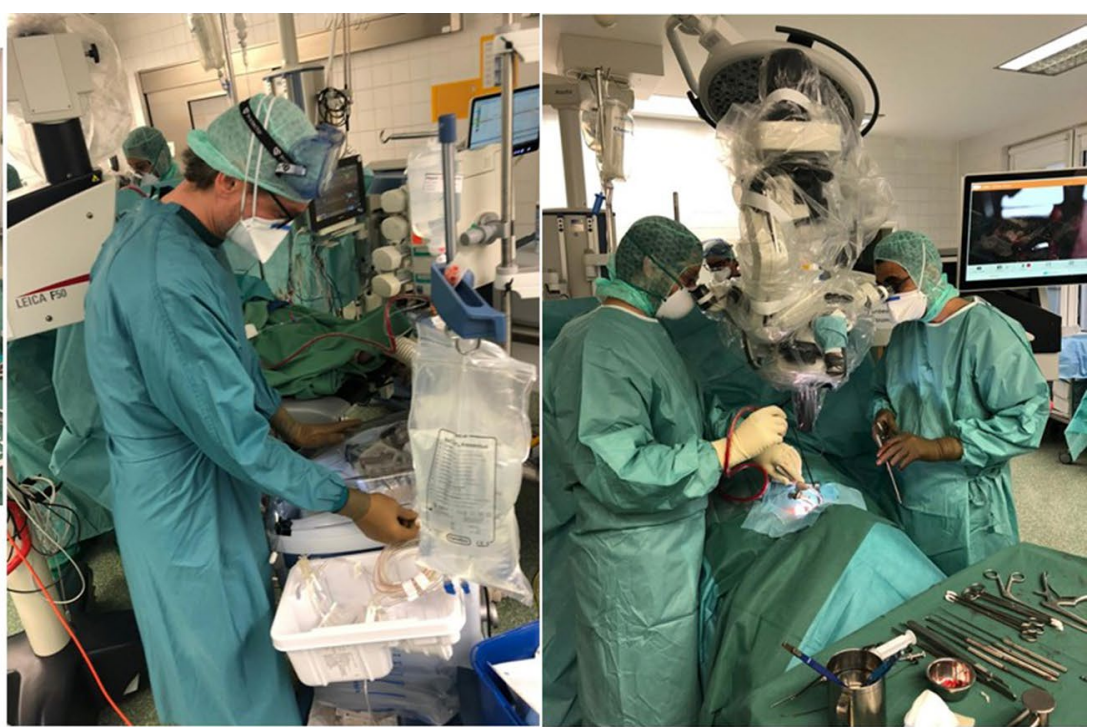

B
C

Fig. 3 Operating room setting during the operation. Surgical team (a), anaesthesia team (b) and the operating room equipment (c) 
the neurological status was recorded (ASIA B). On the first postoperative day ( $12 \mathrm{~h}$ postoperatively), the result of the COVID-19 test was negative. The patient had no respiratory symptoms and was transferred from the suspected region of the Corona ICU to the normal intermediate care unit (IMC) for postoperative observation. The patient was transferred on the third postoperative day to the paraplegia rehabilitation clinic. Two weeks postoperatively, the patient had partial sensory improvement.

\section{Discussion}

\section{Triage during the pandemic}

The COVID-19 outbreak has induced many changes in the practice of spine surgery. Postponement of elective spine surgeries in order to save hospital capacities for COVID-19 patients as well as to prevent nosocomial infections of elective patients was undertaken in our institution. We excluded four categories from the postponement plan, namely: neurological deficits, spondylodiscitis, tumours and fractures. There is a consensus about the importance of such measures among most of the surgeons worldwide [13]. A comparable plan was undertaken by Soh et al. in Singapore, who reduced their elective lists to about $50 \%$ of the norm and postponed deformity and complex revision cases. Donnally et al. published the Rothman Institute guidelines for spine surgery in the COVID-19 era [14]. They recommended triaging spinal operations into three levels. They recommended the surgery in the hospital for level I emergency cases, postponement of surgery for level III elective cases and surgery at ambulatory surgical centres for level II, including degenerative pathologies with intractable pain. In our experience, we underwent an individual discussion with these level II patients with clarification of the possible risks, and when the decision for surgery was taken, this was then performed in the hospital. The same recommendation was given by Jain et al. [15], who again adapted a guide to outlining a triaging system of spine-specific surgical care. The crucial prerequisite to make these guidelines applicable is the availability of adequate healthcare resources to safely perform the procedures.

\section{Operating room considerations}

Dedicated operating theatres for COVID-19 patients are utilized in our hospital. These theatres are well equipped with an operating microscope and two C-arms. This step took place at the very beginning of the pandemic, and the rooms remained unused, waiting only for COVID-19 patients. The same concept has been recommended by Soh et al. [13] as well as Jain et al. [15]. In case of emergency, delaying the surgery waiting for the COVID test is not clinically permissible. The case presented in this work with cervical spine fracture-dislocation reflects the importance of such measures. This plan helps to achieve the best medical service for any patient without any potential risk of increasing virus transmission.

\section{Perspectives for the near future}

Donnally et al. [14] emphasized the fact that surgical delays should not be assumed to be for a short time. The risk of postponing a spine surgery should be assessed, understanding that eventual surgery may occur in 3-4 months later. In our hands, the decision for delaying surgery is taken after a thorough evaluation of the patient's radiological examinations and records as well as after a phone conversation with each patient to get informed about his actual state regarding the spine as well as the accompanying social and medical aspects. Furthermore, the role of telemedicine is getting more critical in our hospital. We increased the number of institutions connected to our department to offer more telemedical service. The online consultation, together with teleradiological connections, became a standard option besides the usual phone calls. Recommendations and regulations regarding the safe use of telemedicine are evolving to ensure both patient data protection and the possibility to minimize the unnecessary visits and patient transfer between hospitals [16].

Once an urgent surgery is performed, we aim to reduce the hospital stay to a minimum to decrease the strain on our hospital beds and healthcare resources. The case presented in this work was operated upon $135 \mathrm{~min}$ after admission and was then transferred postoperatively after three days to the paraplegic rehabilitation clinic, which was also beneficial to the patient to enhance neurological recovery. Soh et al. [13] recommended the same concept in their publication. They highlighted the current climate of economic uncertainty, and that shorter hospital stays would allow keeping healthcare costs down for patients and the nation. We recommend maintaining this strategy even after the pandemic as rapid discharge may reduce the possibility of in-hospital infections and reduce the cost for patients and the medical system.

A last aspect for the future is the extension of telemedicine service to include surgical interventions. Tian et al. [17] recently published their experience with telerobotic spinal surgery based on 5G technology. They performed remote surgery in 12 cases and concluded that this technology could build a connection between surgeons and patients located in isolated or rural areas with a lack of medical resources. We do not have experience with this technology; however, this might be an attractive option in such pandemic situations provided the necessary infrastructures are available. 


\section{Elective spine operations, is it the right time?}

In the last few weeks, the number of new cases is significantly reduced. We started to perform elective surgeries under close communication with the intensive care and anaesthesia departments. The prerequisites are the availability of ICU reserve and the presence of adequate capacity in the Corona unit. In the last few days, new publications are discussing starting the elective operations during the pandemic $[18,19]$. The main recommendations are summarized in this conclusion; elective surgery resumption should be cautious, especially in the area of pandemic focus. Elective surgery should ideally be strictly performed in a COVIDfree facility, and hospital stay should be as short as possible. Patients operated first should be carefully selected according to COVID exposure, age, ASA physical status classification system/risk factors, socio-professional situation and surgical indication. At the suspicion of COVID symptoms, elective surgery should be postponed. In the case of surgery, close monitoring of COVID-19 signs and adapted personal protective equipment is recommended [18].

\section{Conclusion}

The current COVID-19 pandemic requires an extra-ordinary organization of the medical and surgical care of the patients. It is possible to manage a potentially infected patient surgically, but a multidisciplinary plan is necessary to protect other patients and the medical staff.

\section{Compliance with ethical standards}

Conflict of interest On behalf of all authors, the corresponding author states that there is no conflict of interest.

\section{References}

1. Lu H, Stratton CW, Tang YW (2020) Outbreak of pneumonia of unknown etiology in Wuhan, China: the mystery and the miracle. J Med Virol 92(4):401-402. https://doi.org/10.1002/jmv.25678

2. Di Gennaro F, Pizzol D, Marotta C, Antunes M, Racalbuto V, Veronese N, Smith L (2020) Coronavirus diseases (COVID-19) current status and future perspectives: a narrative review. Int J Environ Res Public Heal 17:2690-2696

3. Huang C, Wang Y, Li X, Ren L, Zhao J, Hu Y et al (2020) Clinical features of patients infected with 2019 novel coronavirus in Wuhan, China. Lancet. https://doi.org/10.1016/S0140 $-6736(20) 30183-5$
4. World Health Organization (2020) Coronavirus disease (COVID19) pandemic. https://www.who.int/emergencies/diseases/novel -coronavirus-2019. Accessed 23 Mar 2020

5. Spiegel Wissenschaft (2020) Erster fall des coronavirus in Deutschland bestätigt https://www.spiegel.de/wissenschaft/mediz in/corona-virus-erster-fall-in-deutschland-bestaetigt-a-19843b8d8694-451f-baf7-0189d3356f99. Accessed 22 Apr 2020

6. Durham DP, Casman EA (2012) Incorporating individual healthprotective decisions into disease transmission models: a mathematical framework. J R Soc Interface. https://doi.org/10.1098/ rsif.2011.0325

7. Hufert F, Spiegel M (2020) Coronaviren: von der banalen Erkältung zum schweren Lungenversagen. Monatsschrift Kinderheilkd. https://doi.org/10.1007/s00112-020-00910-2

8. Burchardi H, Schuster HP, Zielmann S (1994) Cost containment: Europe. Germany. N Horiz (Baltimore, Md.) 2(3):364-374

9. Liu Y, Li J, Feng Y (2020) Critical care response to a hospital outbreak of the 2019-nCoV infection in Shenzhen, China. Crit Care. https://doi.org/10.1186/s13054-020-2786-x

10. Zhang L, Liu Y (2020) Potential interventions for novel coronavirus in China: a systematic review. J Med Virol. https://doi. org/10.1002/jmv.25707

11. He F, Deng Y, Li W (2020) Coronavirus disease 2019: What we know? J Med Virol. https://doi.org/10.1002/jmv.25766

12. Shousha M (2014) ABCD classification system: a novel classification for subaxial cervical spine injuries. Spine. https://doi. org/10.1097/BRS.0000000000000257

13. Soh TLT, Ding BTK, Yap WMQ, Oh JY-L (2020) Spine surgery and COVID-19: early experiences from Singapore. Spine. https ://doi.org/10.1097/BRS.0000000000003532

14. Donnally CJ 3rd, Shenoy K, Vaccaro AR, Schroeder GD, Kepler CK (2020) Triaging spine surgery in the COVID-19 era. Clin Spine Surg 33(4):129-130. https://doi.org/10.1097/BSD.00000 00000000988

15. Jain NS, Alluri RK, Schopler SS, Hah R, Wang JC (2020) COVID-19 and spine surgery: a review and evolving recommendations. Glob Spine J. https://doi.org/10.1177/219256822092365 5

16. Garg V, Brewer J (2011) Telemedicine security: a systematic review. J Diabetes Sci Technol 5(3):768-777. https://doi. org/10.1177/193229681100500331

17. Tian W, Fan M, Zeng C, Liu Y, He D, Zhang Q (2020) Telerobotic spinal surgery based on $5 \mathrm{G}$ network: the first 12 cases. Neurospine 17(1):114-120. https://doi.org/10.14245/ns.1938454.227

18. Mouton C, Hirschmann MT, Ollivier M, Seil R, Menetrey J (2020) COVID-19-ESSKA guidelines and recommendations for resuming elective surgery. J Exp Orthop 7(1):28. https://doi. org/10.1186/s40634-020-00248-4

19. Diaz A, Sarac BA, Schoenbrunner AR, Janis JE, Pawlik TM (2020) Elective surgery in the time of COVID-19. Am J Surg S0002-9610(20)30218-X. Advance online publication. https:// doi.org/10.1016/j.amjsurg.2020.04.014

Publisher's Note Springer Nature remains neutral with regard to jurisdictional claims in published maps and institutional affiliations. 